This is a post-peer-review, pre-copyedit version of an article published in Chromosoma. The final authenticated version is available online at: https://doi.org/10.1007/s00412-014-0452-2

\title{
Consolidation of the genetic and cytogenetic maps of turbot (Scophthalmus maximus) using FISH with BAC clones
}

Xoana Taboada • Jose C. Pansonato-Alves • Fausto Foresti • Paulino Martínez • Ana Viñas • Belén G. Pardo • Carmen Bouza

X. Taboada $•$ A.Viñas

Departamento de Genética, Facultad de Biología, CIBUS, Universidad de Santiago de Compostela, 15782Campus Vida, Santiago de Compostela, Spain

J. C. Pansonato-Alves • F. Foresti

Departamento de Morfologia, Universidade Estadual Paulista, Botucatu, Brazil

P. Martínez • B. G. Pardo • C. Bouza $(*)$

Departamento de Genética. Facultad de Veterinaria, Universidad de Santiago de Compostela, 27002 Lugo, Spain

e-mail: mcarmen.bouza@usc.es

\begin{abstract}
Bacterial artificial chromosomes (BAC) have been widely used for fluorescence in situ hybridization (FISH) mapping of chromosome landmarks in different organisms, including a few in teleosts. In this study, we used BAC-FISH to consolidate the previous genetic and cytogenetic maps of the turbot (Scophthalmus maximus), a commercially important pleuronectiform. The maps consisted of 24 linkage groups (LGs) but only 22 chromosomes. All turbot LGs were assigned to specific chromosomes using BAC probes obtained from a turbot $5 \times$ genomic BAC library. It consisted of 46,080 clones with inserts of at least $100 \mathrm{~kb}$ and $<5 \%$ empty vectors. These BAC probes contained gene-derived or anonymous markers, most of them linked to quantitative trait loci (QTL) related to productive traits. BAC clones were mapped by FISH to unique marker-specific chromosomal positions, which showed a notable concordance with previous genetic mapping data. The two metacentric pairs were cytogenetically assigned to LG2 and LG16, and the nucleolar organizer region (NOR)bearing pair was assigned to LG15. Double-color FISH assays enabled the consolidation of the turbot genetic map into 22 linkage groups by merging LG8 with LG18 and LG21 with LG24. In this work, a first generation probe panel of BAC clones anchored to the turbot linkage and cytogenetical map was developed. It is a useful tool for chromosome traceability in turbot, but also relevant in the context of pleuronectiform karyotypes, which often show small hardly identifiable chromosomes. This panel will also be valuable for further integrative genomics of turbot within Pleuronectiformes and teleosts, especially for fine QTL mapping for aquaculture traits, comparative genomics, and whole-genome assembly.
\end{abstract}

Keywords BAC library • BAC-FISH • Genetic map • Cytogenetic map • Turbot

\section{Introduction}

Flatfish (Orden Pleuronectiformes) are cultured worldwide because of their commercial interest. In the Northeast Atlantic area, 11 species important for aquaculture are exploited, turbot (Scophthalmus maximus) production being the highest among European pleuronectiforms (Cerdà and Manchado 2013). This is an appreciated species with a high commercial value and a high potential growth rate under intensive culture conditions. Turbot culture started in the UK and France in the early 1970s. In Spain, turbot farming commenced in the late 1980s, but finally becoming Europe's first turbot producer. It is predicted that in 2014, the total turbot production will duplicate that of 2009 (9,142 t) (Federation of European Aquaculture producers, FEAP 2010). Also, turbot farming has become one of China's main marine cultured species, its production being around 50,000 t per year (Lei and Liu 2011). Current research in this species is mainly focused on controlling sex ratio, increasing growth rate and improving disease resistance (Bouza et al. 2012). Karyotypic analysis in turbot has been carried out using conventional banding techniques (Giemsa, C-banding, Ag- 
staining), fluorochromes, and restriction nuclease-banding. It shows a diploid number of 44 chromosomes and a fundamental number of 48 arms with no sex-associated chromosome heteromorphisms (Bouza et al. 1994; Cuñado et al. 2001; Pardo et al. 2001). As in other pleuronectiform species, the turbot chromosomes are small and with similar sizes, which hinders karyotype ordination and therefore makes it difficult to analyze chromosomes and evolution in this group of teleosts (Pardo et al. 2001). Available data suggest that karyotype evolution in Pleuronectiformes has been dealt with the reduction of their diploid number, mainly due to Robertsonian fusions, and several chromosome inversions (Oliveira et al. 2007). Further information is necessary for a better understanding of the role of chromosome changes in the evolution of this group (Wang et al. 2009), in a context of controversial phylogeny (Azevedo et al. 2008; Campbell et al. 2013). In the age of new generation sequencing (NGS), linkage mapping constitutes an important tool to analyze several genome architecture issues. Genetic maps are essential tools to locate genomic regions associated with productive traits. They can eventually be applied to perform marker-assisted selection programs or to identify specific genes through fine mapping and positional cloning strategies (Danzmann and Gharbi 2001; Canario et al. 2008). Additionally, they support the study of genome organization and evolution through comparative mapping, and provide useful landmarks for genome assembly (Shimoda et al. 1999; Gross et al. 2008; Kai et al. 2011). A gene-enriched turbot linkage map has enabled the genomic investigation in this species, the comparative mapping against model genomes, and also the localization of quantitative trait loci (QTL) and genes related to important commercial traits (sex, growth, and pathogen resistance) (Martínez et al. 2009; Rodríguez-Ramilo et al. 2011; 2013a; 2013b; SánchezMolano et al. 2011; Bouza et al. 2012). Recent compilation of all previous mapping resources of turbot (Bouza et al. 2007; 2012; Martínez et al. 2008; Ruan et al. 2010; Viñas et al. 2012) allowed the construction of an integrated dense consensus map useful to study genome evolution and support genetic breeding strategies in this species (Hermida et al. 2013a). Despite its short intermarker distance, among the highest in density among non-model teleosts (3.7 cM; Hermida et al. 2013a), this map comprises more linkage groups than expected according to its karyotype (24 linkage groups vs. 22 chromosome pairs). Thus, further work is still required for the final consolidation of this turbot map. Bacterial artificial chromosomes (BAC) libraries are essential tools, which have been widely used in many areas of genomics and genetics research, including positional cloning (Zhang et al. 2008), integration of physical and genetic maps (Xu et al. 2007), development of region targeted markers (Xu et al. 2006), and comparative genome analysis (Wang et al. 2007). BAC libraries have been successfully developed for some important teleostean species (Wang et al. 2008; Shao et al. 2010; Xia et al. 2010; Jeukens et al. 2011; Kuhl et al. 2011), but such large-insert genomic DNA libraries have not been reported to date for the turbot. BAC-FISH represents a very powerful technique to localize large DNA inserts along chromosomes. The integration of genetic and cytogenetic maps with sequencing data has provided new marks and information about genome organization and evolution in different organisms, including teleosts. Cytogenetic methodologies have supplied a complementary mapping approach to other genetic and physical mapping strategies for genome assembly in teleosts, to consolidate existing framework maps and for comparative evolutionary analysis (Phillips et al. 2006a; 2006b; 2009; Phillips et al. 2013; Freeman et al. 2007; Sasaki et al. 2007; Guyon et al. 2012; Mazzuchelli et al. 2012; GarcíaCegarra et al. 2013). The objective of this study was the physical assignment of the 24 linkage groups of the turbot genetic map to the 22 chromosome pairs of its karyotype, using as FISH probes BAC clones containing molecular markers mapped into specific linkage groups (LGs). The purpose is to integrate the linkage and cytogenetic maps of the turbot, providing physical and genetic anchors to be combined with the increasing amount of genomic data in this species. The chromosome-specific clones will constitute valuable cytogenetic markers for structural and evolutionary genomic research in the turbot, and for a better understanding of the chromosome evolution of Pleuronectiformes.

\section{Material and methods}

\section{BAC library characterization}

The turbot BAC library was generated at the Clemson University Genomics Institute (CUGI) under ReGABA (Galician Network of Biotechnology in Aquaculture) sponsorship. The library was constructed from high molecular weight nuclear DNA of a highly inbred S. maximus single female. The size-selected high molecular weight DNA fragments from the 100- to 350-kb size fraction were ligated into the pIndigoBAC536 BAC vector. Ligation products were trans formed into Escherichia coli DH10B electrocompetent cells. To evaluate the average size of inserted fragments in the library, 96 BAC clones were randomly picked and their plasmids isolated by alkaline-SDS method, inserts released with a rare cutter enzyme, and finally, separated from the vector by pulsed field gel electrophoresis (PFGE).

\section{BAC library screening}

The most recent consensus linkage map of turbot (Hermida et al. 2013a) contained 487 single nucleotide polymorphism (SNP) and microsatellite markers, $40 \%$ linked to expressed sequence tags (EST), which clustered at 24 
linkage groups. This map showed a total length of $1,414 \mathrm{cM}$ with low intermarker distance $(3.3 \mathrm{cM})$ and high genomic coverage $(\approx 90$ $\%$ ). To isolate BAC clones for each turbot linkage group (LG1-LG24), we screened the library with primers from a selection of anchor markers based on previous map information, which includes the centromere mapping positions (Martínez et al. 2008; Hermida et al. 2013a). A total of 37 markers were selected across the turbot genetic map (1-3 markers per linkage group; Supp-Fig. S1) based on their map position, functional annotation (Bouza et al. 2012; Hermida et al. 2013a) and their association with QTLs related to productive traits (Martínez et al. 2009; Rodríguez-Ramilo et al. 2011; 2013a; 2013b; Sánchez-Molano et al. 2011; Viñas et al. 2012). Allelic size was also considered to optimize the multiplex polymerase chain reaction (PCR) screening when possible. Most markers were EST-derived ( $81 \%$; 4 SNP and 26 microsatellites), but seven anonymous markers were also selected at relevant regions of the genetic map lacking gene markers, such as the smallest LG24 or within the confidence interval of some QTLs (growth at LG12 and LG14; disease resistance at LG23; Table 1). We chose at least one marker per linkage group to establish the correspondence between the genetic (24 LGs) and cytogenetic $(n=22)$ maps. BAC library screening was carried out to find at least one positive BAC clone among the selected markers for each linkage group of the turbot map. A two-step polymerase chain reaction (superpools PCR and 3D (dimension)-PCR; Asakawa et al. 1997) with slight modifications was carried out to maximize the effectiveness of BAC library screening. Multidimensional BAC pools were generated and DNA was extracted by alkaline-SDS method. Briefly, we divided the library evenly in two parts, 60 plates of 384 clones each one. Each of these parts was split into ten superpools (SP) consisting of $6 \times 384$ plates each. The first PCR step was applied to identify positive SP for each selected marker. Then, the second step PCR screening was used to identify individual BAC clones by 3D-PCR procedure: (1D) to identify the positive plate/s among the six ones of the positive SP; (2D) to identify the positive column/s among the 24 mixtures in which BAC-DNA clones of each column of the six plates of the SP are mixed; and (3D) to identify positive raw/s among 16 mixtures (BAC-DNA clones of each raw of the six plates per SP). This two-step pooling scheme reduces the number of PCR assays to $56(10+6+24+16)$ for the identification and isolation of positive clones for each linkage group anchor marker in the first part of the library. If no positive clone was identified, then the second part of the library was screened. PCR products for all markers and hierarchical multidimensional BAC pools were checked using a positive PCR control of genomic DNA on $2 \%$ agarose gels or multiplexed on an automatic sequencer ABI3730 (Applied Biosystems).

\section{Chromosome preparation and FISH procedure}

The chromosomal preparations were obtained from anterior kidney cells after in vivo colchicina treatment, following the techniques described by Foresti et al. (1981) and Bouza et al. (1994), starting from ten juvenile specimens of turbot (around $80 \mathrm{~g}$ ) sampled in the research facilities of the Cluster de Acuicultura de Galicia (CETGA). Twenty-eight BAC clones were finally labeled with biotin- 16dUTP or digoxigenin-11-dUTP (Roche Applied Sciences) using whole-genome amplification kits, WGA2 and WGA3 (Sigma-Aldrich), according to the supplier's protocol. In addition, a 28S rDNA probe was directly labeled with biotin-16-dUTP (Roche Applied Sciences) by PCR (Forward/Reverse primers: 5'-ACCCGCTGAATTTAAGCAT-3'/5'TGAACT CTCTCTTCAAAGTTCTTTTC-3') following the protocol described by Chen et al. (2003) with slight modifications. The FISH procedure was carried out according to the procedure established by Pinkel et al. (1986) with some modifications described below. The hybridization mixture $(60 \mu l$ for each slide) contained $5 \mathrm{ng} / \mu \mathrm{l}$ of each labeled BAC/28S probe, $50 \%$ formamide, $10 \%$ dextran sulfate, $2 \times \mathrm{SSC}$, and $50 \mathrm{ng} / \mu \mathrm{l}$ of salmon sperm DNA (Invitrogen). These probe cocktails were denatured at $100^{\circ} \mathrm{C}$ for $10 \mathrm{~min}$, added to the slides, and incubated at $37{ }^{\circ} \mathrm{C}$ overnight. Biotin and digoxigenin-labeled probes were detected using avidin-FITC (Invitrogen) and antidigoxigenin rhodamine (Roche Applied Sciences), respectively. Slides were counterstained with DAPI/Vectashield (Vector Laboratories) and photographed with Olympus BX51 microscope equipped with an Olympus DP71 color digital camera. Digital images were imported into Adobe Photoshop version 3.0 for final processing. This program was also used to perform chromosomal measurements. The relative chromosome sizes were calculated using the largest metacentric chromosome assigned to LG2 by FISH- BAC as an internal standard, and measurements for each LG-specific chromosome averaged over four metaphases.

\section{Results and discussion}

\section{BAC library characterization and screening}

BAC libraries are critical for identifying full-length genomic sequences to integrate genetic and physical maps (Yim et al. 2007; Wang et al. 2008; Ninwichian et al. 2012; Zhao et al. 2013), and for comparative genomics studies (Maughan et al. 2009; Kuhl et al. 2010). Traditionally, they have been the first step towards whole-genome sequencing projects (Monaco and Larin 1994; Palti et al. 2011). In this study, the first BAC genomic library of turbot (S. maximus) was characterized. It consisted of a total of 46,080 clones, which were arrayed in 120 plates of 384 -microtiter wells. To evaluate the 
Table 1 Information on the markers selected for screening the turbot genomic library and the BAC clones used as probes for FISH

\begin{tabular}{|c|c|c|c|c|c|}
\hline LG & Marker & Accession number ${ }^{\mathrm{a}}$ & Turbot BAC clone & Functional annotation $^{\mathrm{a}}$ & $\mathrm{QTL}^{\mathrm{b}} /$ centromere mapping $^{\mathrm{c}}$ \\
\hline 1 & SmaUSC-E15 & FE943893 & Sma38.I23 & Non annotated cDNA & G-QTL interval (close to SmaUSC15, $14 \%$ VPE) \\
\hline 2.1 & Sox 19 & JQ403639 & Sma102.M5 & Sox19 & V-QTL interval (close to SmaUSC168, 7 \% VPE) \\
\hline 2.2 & Sma-USC187 & DQ810987 & Sma33.E1 & Anonymous & Close to centromere-linked marker (SmaUSC109) \\
\hline 3 & Sma-E118 & HS031242 & Sma47.E7 & UBR1 & P-QTL-marker (close to SmaUSC200, 8 \% VPE) \\
\hline 4 & cyp19a & JQ403643 & Sma50.D3 & Cyp19a & P-QTL interval \\
\hline 6 & SmaUSC-E29 & FE946649 & Sma33.C21 & Non annotated cDNA & V-QTL interval (close to SmaUSC110, $14 \%$ VPE) \\
\hline 7 & Sma-E100 & HS029651 & Sma52.010 & $S H 2 D 1 A$ & P-QTL interval \\
\hline 8 & SmaUSC-E43 & FE945769 & Sma54.M1 & MIRN144 & V-QTL interval \\
\hline 9 & SmaUSC-E23 & FE945024 & Sma56.G24 & $\mathrm{BDH} 2$ & Associated to P-QTL and A-QTL ( $8 \%$ and $12 \%$ VPE) \\
\hline 10 & Sma-E290 & DQ810879.1 & Sma43.G3 & PCLB1 & Close to centromere-linked marker (SmaUSC79) \\
\hline 14.1 & SmaUSC-E28 & FE94619 & Sma39.D18 & $P A 2 G 4 B$ & G-QTL interval \\
\hline 14.2 & SmaUSC-82 & DQ810882 & Sma33.K8 & Anonymous & Associated to G-QTL (11 \% VPE) \\
\hline 15 & SmaUSC-E8 & FE946444 & Sma54.I11 & GAL-9 & G-QTL interval \\
\hline 16.1 & Sma-E158 & EY455452.1 & Sma48.N17 & CLCN5 & G-QTL interval \\
\hline 16.2 & Sma-E137 & EY454995.1 & Sma51.E1 & $F G A$ & G-QTL interval \\
\hline 16.3 & Sma-E183 & HS029459 & Sma50.B11 & BTG1 & G-QTL interval \\
\hline 17 & Sma-E112 & HS030447 & Sma28.M16 & $V I L 1$ & V-QTL (close to SmaUSC91, $10 \%$ VPE) \\
\hline 18 & SmaUSC-E19 & FE944607 & Sma46.L24 & Non annotated cDNA & G-QTL interval \\
\hline 19 & Sma-E205 & FE945305.1 & Sma27.N21 & GALM & G-QTL interval \\
\hline
\end{tabular}

${ }^{\text {a }}$ Functional annotation of markers in the integrated linkage map of turbot (Hermida et al. 2013a)

${ }^{\mathrm{b}}$ Turbot QTL information for growth (G), sex determination (SD), and disease resistance (Philasterides dicentrarchi-P; Aeromonas salmonicida-A and VHS virus-V) traits. Most markers were mapped within previously reported QTL intervals or close to other markers significantly associated with the evaluated traits, or significantly associated themselves (Martínez et al. 2009; Sánchez-Molano et al. 2011; Rodríguez-Ramilo et al. 2011; 2013a; 2013b). VPE (\%): percentage of phenotypic variance explained by significantly associated markers to productive traits

${ }^{\mathrm{c}}$ Centromere mapping information for some selected markers, following Martínez et al. (2008)

average size of the inserted fragments in the library, DNA was prepared from 96 randomly selected recombinant BAC clones. After digestion and separation of the fragments by PFGE, it was possible to resolve the vector DNA band (7.5 kb) and one or more bands of the insert depending on the number of restriction sites in the cloned insert. The library included inserts of at least $100 \mathrm{~kb}$ and $<5 \%$ non-recombinant or empty vectors. The library provided fivefold coverage of the haploid genome of the species, based on the estimated genome size of turbot ( $800 \mathrm{Mb}$; Cuñado et al. 2002; Bouza et al. 2007). Integration of physical and genetic maps can be done by placing and ordering BAC clones and contigs along a genetic map (Paux et al. 2008; You et al. 2010). For this, BAC library screening is an essential step. Until few years ago anchoring was a rate-limiting step mainly due to technical limitations in screening and also to the lack of high quality dense genetic maps. In this study, primers for a selected panel of anonymous and gene markers mapped to each linkage group of the turbot genetic map (Hermida et al. 2013a) were successfully used for two-step PCR-based screening of the BAC library constructed in this species, as previously reported in other farmed teleosts (Wang et al. 2008). This approach represents a useful low-cost alternative and enhances efficiency regarding classical hybridization-based screening (Asakawa et al. 1997; Yim et al. 2007). This procedure reduces the number of PCR assays and decreases the number of false-positives (Yim et al. 2007; Luo et al. 2009; You et al. 2010). 
The screening involved 37 selected markers, and most of them ( $84 \%$; Online Resource 1) were positively amplified in at least one BAC clone, which demonstrates a notable genome coverage of the turbot BAC library. A panel of positive marker-specific BAC clones was selected for further isolation and labeling to carry out FISH. Only one marker revealed multiple BAC clones in the two-step PCR screening likely associated to repetitive DNA (Sma-E71 at LG9). No positive BAC clones were found for six microsatellite markers at different linkage groups (Sma-E277 at LG1; SmaUSC-E30 at LG5; Sma-E248 at LG9; Sma-E184 at LG17; Sma-E127 and SmaUSC-E33 at LG23; Online Resource 1). It suggests that coverage of the BAC library was randomly distributed across the genetic map. The lack of amplification of the closely linked marker to the major sex-as sociated QTL in turbot (SmaUSC-E30; Martínez et al. 2009) was particularly disappointing. Thus, other markers derived from fine mapping strategies will be required for physical characterization of this region in the turbot library. A final panel of 28 positive marker-specific BAC clones (Table 1) was selected for further isolation and labeling to carry out FISH for chromosomal assignment of the 24 linkage groups of the turbot genetic map. The selection was performed considering two arms at LG2 and LG16 according to their centromere positions (Martínez et al. 2008). In addition, some markers significantly associated to QTL and/or within QTL confidence intervals for growth, disease resistance, and sex determination were also selected (Martínez et al. 2009; Rodríguez-Ramilo et al. 2011; 2013a; 2013b; Sánchez-Molano et al. 2011). Thus, these BAC clones can also be used for fine physical mapping of relevant genomic regions for turbot production by shotgun sequencing using different NGS methods. The efficiency of the two-step PCR screening for the 28 effective markers revealed a variable number of positive superpools in the first PCR step (ranging from 1 to 9; 3.2 on average). After the second step of the PCR-based screening, 1 to 4 positive clones were finally identified per superpool (1.5 on average), which yielded a rough coverage estimate of 4.8 , close to the theoretical expectation of genome coverage (5×). Accordingly, the turbot BAC library represents a very useful genomic resource for physical mapping, positional cloning, comparative genomic studies, and genome sequencing assembly. Assignment of linkage groups to chromosomes: Consolidation of the turbot genetic map. In this report, the 24 linkage groups of the turbot genetic map were assigned to specific chromosomes using FISH with BAC probes, many containing functionally relevant markers mapped to each linkage group (Table 1). Most BAC clones were cytogenetically mapped by one-color FISH to a unique chromosomal position. Three clear bi-armed chromosome pairs were identified and cytogenetically mapped in this study, in agreement with previously reported features of the turbot karyotype (Bouza et al. 1994; Pardo et al. 2001; Cuñado et al. 2002). The two metacentric pairs of different size were assigned to LG2 and LG16 (large and small chromosome pairs, respectively; Fig. 1). The NOR-bearing submetacentric pair was anchored to the LG15 by two-color FISH assay (Fig. 2), which represents the bi-armed chromosome 3 in the turbot karyotype (Bouza et al. 1994; Pardo et al. 2001). Despite the fact that the remaining 19 subtelo-or acrocentric pairs were hardly distinguished by morphology and size, as previously reported (Pardo et al. 2001), in this study, they could be unambiguously assigned to specific linkage groups of the turbot genetic map (Fig. 1). Among them, the major sex determination QTL-bearing chromosome (LG5; Martínez et al. 2009) was associated with the candidate sexual pair of turbot. It will be useful for further sex-related cytogenetic and genomic research using enriched libraries derived from chromosomal micro-dissection. FISH using chromosome-specific probes has proven to be a useful cytogenetic tool to study the origin of sex chromosomes in teleostean species (Wang et al. 2009; Cioffi et al. 2011). Furthermore, double FISH assays in our study enabled us the consolidation of the most dense turbot genetic map consisting of 24 linkage groups (Hermida et al. 2013a) in accordance to the haploid karyotype of the species $(n=22$; Bouza et al. 1994). The present cytogenetic study confirmed the fusion LG8+ LG18, as previously suggested by linkage and comparative mapping evidences (Bouza et al. 2012), and also merged LG21 and LG24 into a single chromosome (Fig. 1). These results reinforce the essential role of the BAC-FISH approach for integrative genome mapping purposes, as reported in other teleosts with even highly dense linkage maps (Brenna-Hansen et al. 2012; Guyon et al. 2012). A good correspondence between genetic and chromosome mapping was revealed by comparing the chromosomal hybridization sites and the respective marker location on the linkage map (Hermida et al. 2013a). Hybridization signals were also mostly in accordance with the centromere positions inferred by the most closely linked markers (Martínez et al. 2008). Using the cytogenetical convention, short arms and/or centromeres were north-oriented for all chromosomes. Thus, the orientation of some LG(LG1, LG3, LG4, LG7, LG8, LG9, LG10, LG11, LG14, and LG22; Fig. 1) had to be inverted in relation to the map order previously published (Hermida et al. 2013a; Online Resource 1). For instance, the LG14 was reversed (Fig. 1) with respect to the previous map position of the centromerelinked marker (SmaUSC253) and hence of the two BAC probes used for FISH (SmaUSC82 in green and SmaUSC-E28 in red) (located at 56.1, 25.2, and $3.7 \mathrm{cM}$, respectively; Online Resource 1). The concordance between FISH and linkage mapping was noteworthy in some cases, such as at LG6 and LG13, which showed an interstitial hybridization site at their correspondent chromosomes, and 
LG1

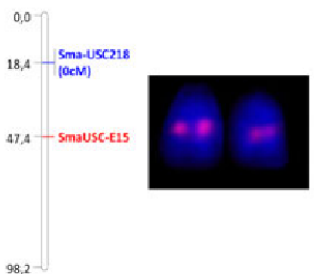

LG5
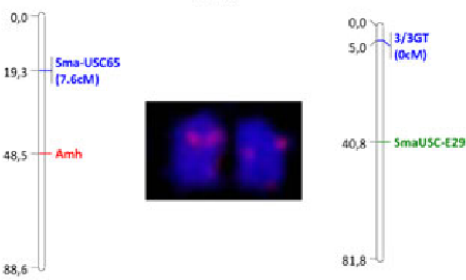

LG9

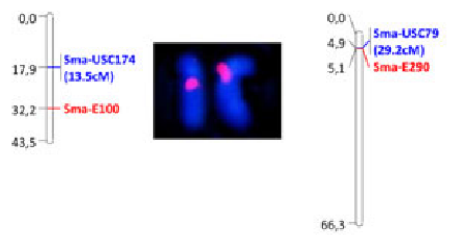

(16,4

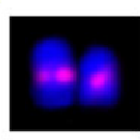

LG17

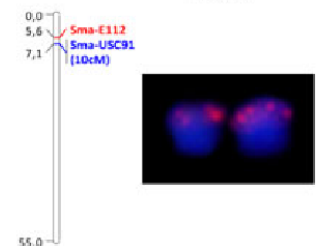

LG22

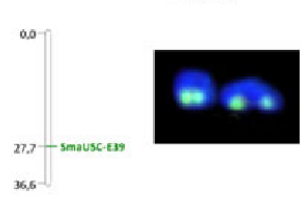

LG2

LG3

LG4
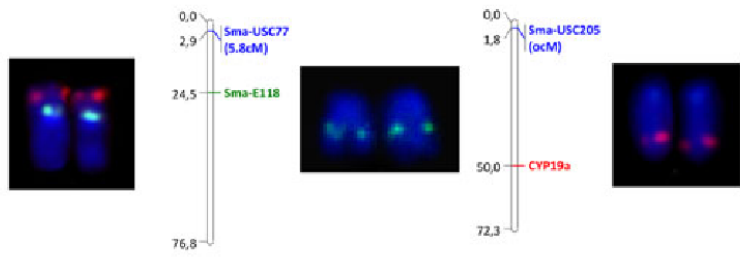

LG6

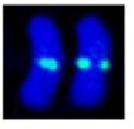

LG10
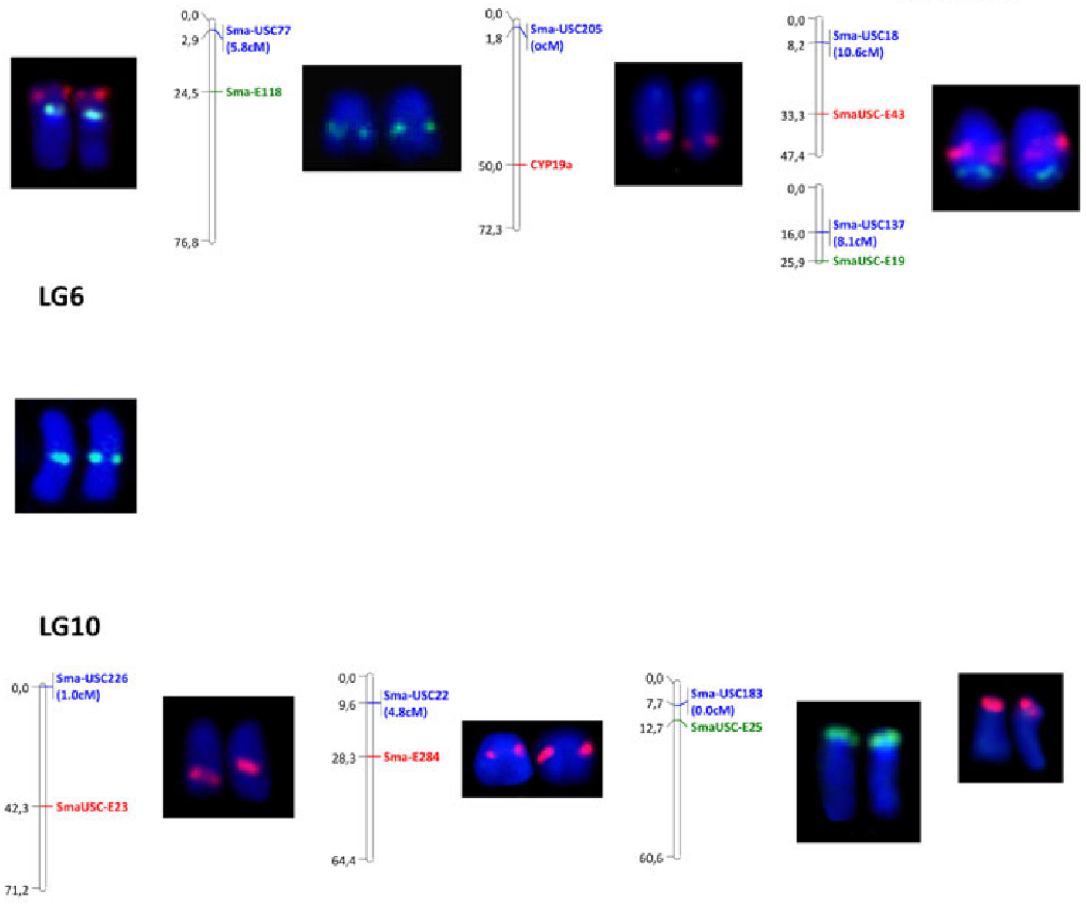
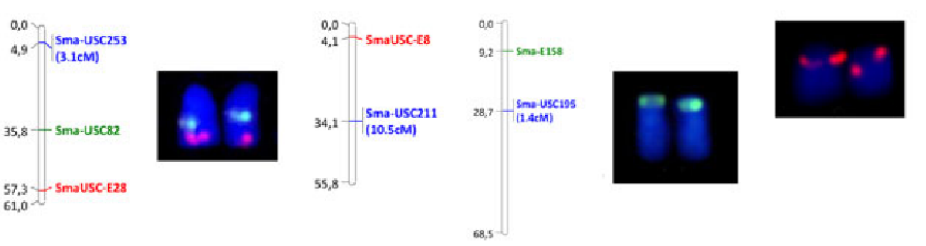

LG19

LG20

LG21+LG24
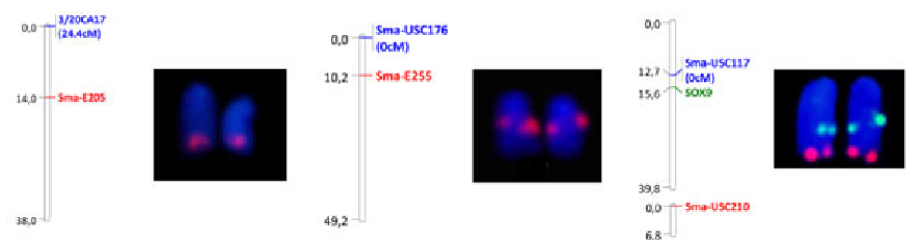

LG23
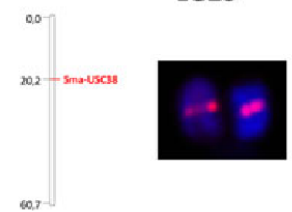

Fig. 1 Assignment of linkage groups to chromosomes of turbot using BAC-FISH. For each chromosome, the linkage group and the selected markers of the genetic map used for BAC library screening (Hermida et al. 2013a) are indicated. Red and green signals correspond to digoxigenin- and biotin-labeled probes, respectively. A closely linked marker to the centromere, with their respective genetic distances within

brackets according to Martínez et al. (2008), is indicated in blue. In some linkage groups, a reverse orientation is presented with regard to the previously published linkage map (Hermida et al. 2013a; see Online Resource 1) to accommodate to karyotype representation with the centromere and/or short arm upwards (LG1, LG3, LG4, LG7-LG1 1, LG14, and LG22) 

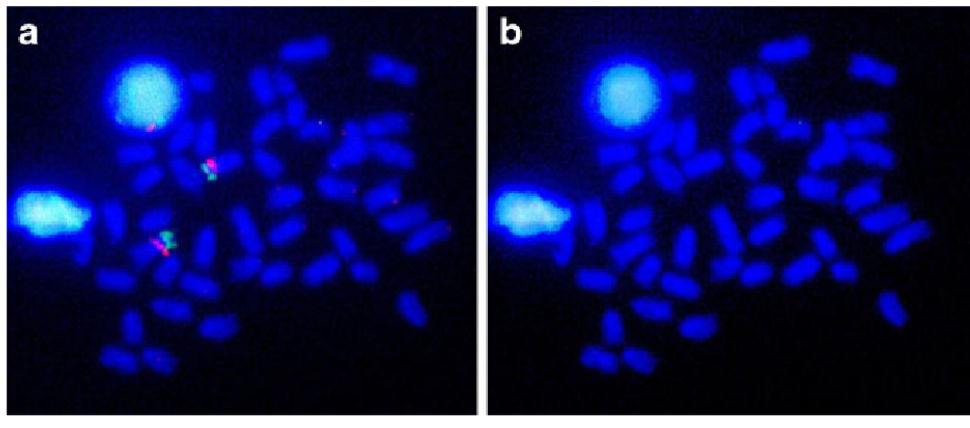

LG16

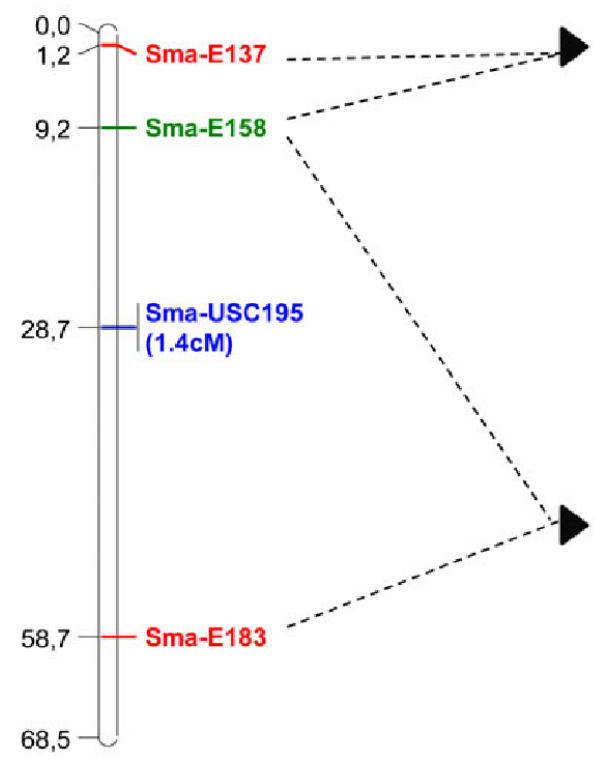

also at LG2 and LG15 with a clear peri/centromeric signal, in agreement with the linkage map position of their respective markers and centromeres (Martínez et al. 2008; Hermida et al. 2013a). Using dual BAC-FISH assays, it was possible to display pairs of markers at the same (Fig. 3a) or alternative (Fig. 3b) chromosomal arms regarding the centromere position of the bi-armed chromosome pair assigned to LG16. Some discrepancies between the marker position in the linkage and cytogenetic maps (LG7, LG19, and LG20) may be partly due to the limitations of linkage mapping of centromeres and their closest markers (up

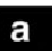

a
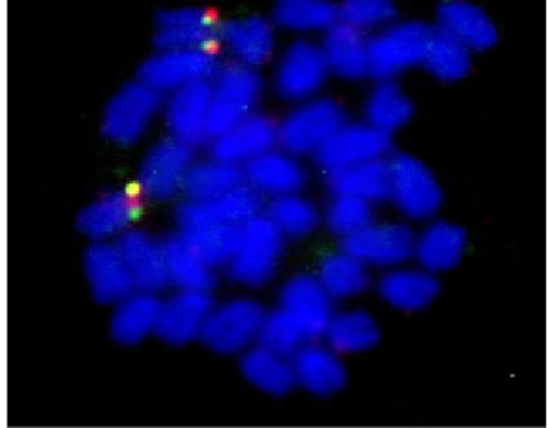

b

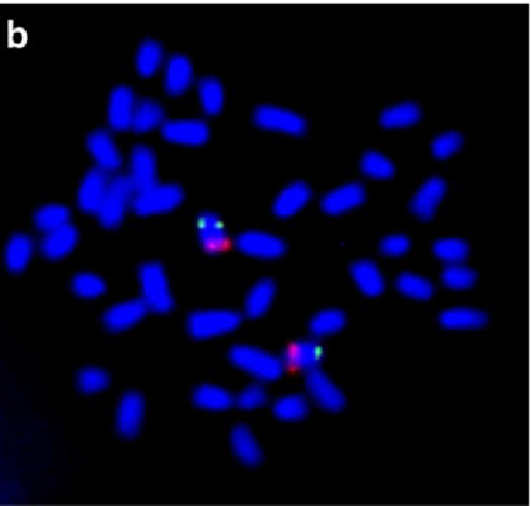
to $13.5 \mathrm{cM}$ distant for LG17) available in turbot to date (Martínez et al. 2008). It could be also related to higher recombination in small acrocentric chromosomes in order to ensure one crossover per chromosome arm, as reported in other teleosts (Phillips et al. 2006a). The assignment of linkage groups to chromosomes by single and double hybridization experiments allowed the direct comparison between physical and genetic distances at chromosome level (Table 2). It was particularly focused on the centromeric position and the relative chromosome sizes, as the two main karyotypic features. Overall, the relative ordination by chromosome size in the turbot karyotype and the linkage group in the species' genetic map were highly concordant (Fig. 4a; Spearman correlation; $r=0.72 ; \mathrm{P}<0.001)$. Thus, the largest and smallest linkage groups were mostly assigned to large (LG1, LG2, LG4, LG5, LG6, LG7, LG8+ LG18, and LG9) and small (LG16-20 and LG22)

Fig. 2 a Identification of the NOR-bearing chromosome pair by double hybridization using the BAC-clone Sma54.I11 for LG15 and 28S rDNA probes, labeled with digoxigenin (red) and biotin (green), respectively, b reference metaphase without signals

Fig. 3 Double hybridization assays on chromosome-LG16. Markers labeled in red and green in the left representation were used to screen the BAC library. a Dual FISH assay using BAC clones for closely linked markers in the short arm of LG16 (Sma- E137 in red and Sma-E158 in green), b Dual FISH assay using BAC clones at each arm of LG16 (Sma-E158 at LG16p in green and Sma-E183 at LG16q in red) 

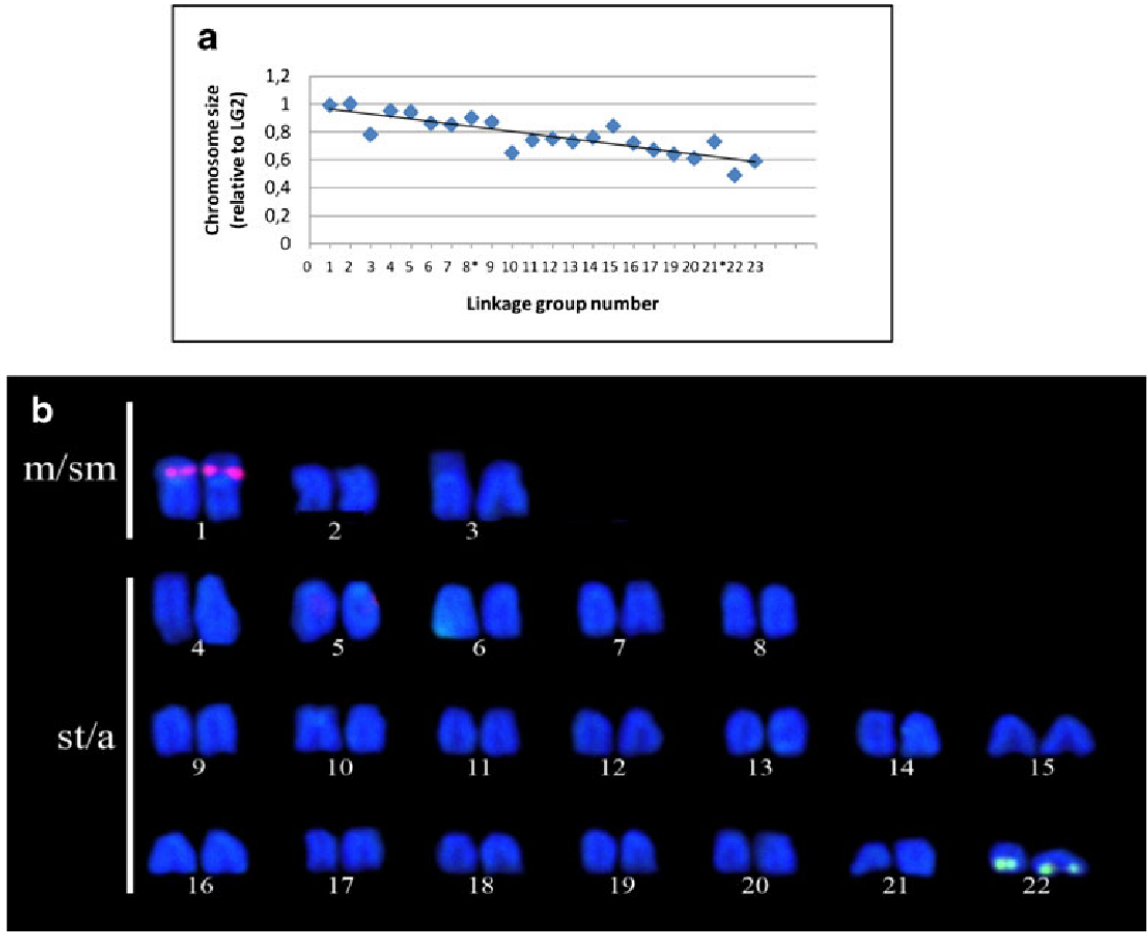

Table 2 Relative size of turbot chromosomes and of the corresponding linkage groups in the turbot genetic map (Hermida et al. 2013a)

\begin{tabular}{lll}
\hline LG & Relative size (total/LG2) & LG size (cM) \\
\hline 1 & 0.99 & 98.2 \\
2 & 1 & 81.3 \\
3 & 0.78 & 78.8 \\
4 & 0.95 & 72.3 \\
5 & 0.94 & 88.6 \\
6 & 0.86 & 81.8 \\
7 & 0.85 & 43.5 \\
$8+18$ & 0.90 & 73.3 \\
9 & 0.87 & 71.2 \\
10 & 0.65 & 66.3 \\
11 & 0.74 & 64.4 \\
12 & 0.75 & 60.6 \\
13 & 0.73 & 61.8 \\
14 & 0.76 & 61 \\
15 & 0.84 & 55.8 \\
16 & 0.72 & 68.5 \\
17 & 0.67 & 55 \\
19 & 0.64 & 38 \\
20 & 0.61 & 49.2 \\
$21+24$ & 0.73 & 46.6 \\
22 & 0.49 & 36.6 \\
23 & 0.59 & 60.7 \\
\hline & &
\end{tabular}

chromosomes of the karyotype, respectively. Double FISH assays confirmed the cytogenetic identity of LG2 and LG22, respectively, assigned to the highest metacentric and the smallest acrocentric chromosomes in the turbot karyotype (Bouza et al. 1994), thus providing useful references when analyzing metaphase plates (Fig. 4b). LG21 was mapped to a medium-large chromosome as expected, since it merged with LG24. Other minor discrepancies affected to LG3 and LG10 assigned to medium-small chromosomes, and, reversely, to LG23 corresponding to a medium size chromosome. These discordances may be partially related to the maintenance of the original ordination of most linkage groups (Bouza et al. 2007; Martínez et al. 2008) in the consolidated genetic map by Hermida et al. (2013a). They may also be related to the variation in recombination frequency among linkage groups and sexes (Bouza et al. 2012), and thus in the relationship between physical and mapping distances. Discordant size of LG15, which was assigned to the submetacentric NOR-bearing chromosome, is likely related to the reported NOR size polymorphism at its short arm and to the decondensation state of the NORassociated chromatin (Pardo et al. 2001). This work has relevant implications for karyotypic studies in Pleuronectiformes, mostly consisting of small acrocentric chromosomes difficult to differentiate by size. Due to the lack of resolutive chromosome banding in the turbot (Bouza et al. 1994; Pardo et al. 2001), the panel of BAC probes represents a useful tool for identification of individual chromosomes. $\mathrm{We}$

Fig. 4 a Relationship between the length of linkage groups of the turbot genetic map and the correspondent relative sizes of assigned chromosomes by BACFISH. LG18 and LG24 are not represented because they were merged, respectively, with LG8 and LG21 (indicated with asterisks); b Double FISH assays to identify the largest (LG2) and the smallest (LG22) chromosomes of the turbot karyotype, following the main features according to previous cytogenetic data (Bouza et al. 1994; Pardo et al. 2001) 
propose from now on to designate each chromosome pair with a code based upon syntenic markers per linkage group, in order to get a consensus karyotype for the turbot community. For example, markers on LG2 have been cytogenetically mapped to a particular chromosome (the largest metacentric one; Figs. 1 and 4b), which could be now designated as chromosome-LG2, as suggested in zebrafish (Freeman et al. 2007). Moreover, the existence of conserved syntenic patterns between the turbot genetic map and the genomes of model teleosts (Bouza et al. 2012) would allow to investigate the chromosomal relationship between turbot and teleostean karyotypes on the basis of comparative mapping data (Guyon et al. 2012). For example, the relationship between zebrafish chromosomeLG4 (Freeman et al. 2007) and turbot chromosome-LG16, established by a common set of syntenic markers (Bouza et al. 2012), shared similar bi-armed morphology. This comparative mapping approach will be particularly useful for investigating karyotype evolution of Pleuronectiformes (Pardo et al. 2001; Oliveira et al. 2007; García-Segarra et al. 2013). The integration of genetic and chromosomal data would allow to identify orthologous chromosomal regions in others species (Wang et al. 2009; Urton et al. 2011; Mazzuchelli et al. 2012). Interespecific BAC-FISH using turbot probes could be another promising strategy to investigate chromosome evolution among pleuronectiforms, as recently reported in other teleosts (Mazzuchelli et al. 2012; Phillips et al. 2014). It may be tested against related species, with linkage maps based on common markers with the turbot, such as brill (Scophthalmus rhombus) and sole (Solea spp.) (Pardo et al. 2001; Molina-Luzón et al. 2012; Hermida et al. 2013b). The integration of genetic and cytogenetic maps paves the way for a large number of applications (Phillips et al. 2006a; 2006b; 2009; Kuhl et al. 2011; Zhao et al. 2013), which includes the quick assignment of novel markers to a linkage group without previous genetic mapping in segregant populations. In addition, the turbot genetic map and its associated BAC-FISH map provide a valuable resource to study relevant QTL-bearing chromosomes for sex determination, growth, or disease resistance (Martínez et al. 2009; Rodríguez-Ramilo et al. 2011; 2013a; 2013b; Sánchez-Molano et al. 2011). This will allow to explore chromosome polymorphisms between sexes and populations, to characterize relevant BAC clones by NGS methods or to dissect productive complex traits (Ross and Peichel 2008; Kuhl et al. 2011; Xu et al. 2011; Mazzuchelli et al. 2012; Zhao et al. 2013; García-Cegarra et al. 2013). Finally, the integrative mapping represents a support for assembling the forthcoming turbot genome, as recently reported in other teleosts (Lorenz et al. 2010; Brenna-Hansen et al. 2012).

Acknowledgments This study was supported by Spain's Ministerio de Ciencia e Innovación (AGL2009-13273), Consolider Ingenio Aq uag eno mi cs (C SD20 0700002 ) an d Xu nta de Galici a

(09MMA011261PR; 10MMA200027PR). Samples for cytogenetic anal- ysis were kindly supplied by Cluster de Acuicultura de Galicia. Thanks to Víctor González, Manuel Manchado, and Miguel Hermida for technical support and to María López and Vanessa Pérez for technical assistance. We also thank José Antonio Álvarez-Dios for useful comments on the manuscript. The authors wish to acknowledge to the Department of Biología Celular y Ecología of USC for providing the microscope. Finally, the authors are grateful to the people of the laboratory of Dr. Foresti in Botucatu (Brasil) for their technical help with FISH.

\section{References}

Asakawa S, Abe I, Kudoh Y, Kishi N, Wang Y, Kubota R, Kudoh J, Kawasaki K, Minoshima S, Shimizu N (1997) Human BAC library: construction and rapid screening. Gene 19:69-79. doi:10.1016/ S0378-1119(97)00044-9 Azevedo MFC, Oliveira C, Pardo BG, Martínez P, Foresti F (2008) Phylogenetic analysis of the order Pleuronectiformes (Teleostei) based on sequences of 12S and 16S mitochondrial genes. Genet Mol Biol 31:284-292. doi:10.1590/S141547572008000200023 Bouza C, Sánchez L, Martnez P (1994) Karyotypic characterization of turbot (Scophthalmus maximus) with conventional, fluorochrome and restriction endonuclease-banding techniques. Mar Biol 120: 609-613. doi:10.1007/BF00350082 Bouza C, Hermida M, Pardo BG, Fernández C, Fortes GG, Castro J, Sánchez L, Presa P, Pérez M, Sanjuán A, de Carlos A, Alvarez-Dios JA, Ezcurra S, Cal RM, Piferrer F, Martínez P (2007) A microsat- ellite genetic map of the turbot (Scophthalmus maximus). Genetics 177:2457-2467. doi:10.1534/genetics.107.075416 Bouza C, Hermida M, Pardo BG, Vera M, Fernández C, de la Herrán R, Navajas-Pérez R, Álvarez-Dios JA, Gómez-Tato A, Martínez P (2012) An Expressed Sequence Tag (EST)enriched genetic map of turbot (Scophthalmus maximus): a useful framework for compar- ative genomics across model and farmed teleosts. BMC Genet 13: 54. doi:10.1186/1471-2156-13-54 Brenna-Hansen S, Li J, Kent MP, Boulding EG, Dominik S, Davidson WS, Lien S (2012) Chromosomal differences between European and North American Atlantic salmon discov- ered by linkage mapping and supported by fluorescence in situ hybridization analysis. BMC Genomics 13:432. doi:10. 1186/1471-2164-13-432 Campbell MA, Chen W-J, López JA (2013) Are flatfishes (Pleuronectiformes) monophyletic? Mol Phylogenet Evol 69:664-

673. doi:10.1016/j.ympev.2013.07.011 Canario A, Bargelloni L, Volckaert F, Houston RD, Massault C, Guiguen Y (2008) Genomics toolbox for farmed fish. Rev Fish Sci 16:1-13. doi:10.1080/10641260802319479 Cerdà J, Manchado M (2013) Advances in genomics for flatfish aqua- culture. Genes Nutr 8:5-17. doi:10.1007/s12263-012-0312-8 Chen WJ, Bonillo C, Lecointre G (2003) Repeatability of clades as a criterion of reliability: a case study for molecular phylogeny of Acanthomorpha (Teleostei) with larger number of taxa. Mol Phylogenet Evol 26:262-288. doi:10.1016/S1055-7903(02)00371-8 Cioffi MB, Sánchez A, Marchal JA, Kosyakova N, Liehr T, Trifonov V, Bertollo LA (2011) Cross-species chromosome painting tracks the independent origin of multiple sex chromosomes in two cofamiliar Erythrinidae fishes. BMC Evol Biol 11:186. doi:10.1186/1471- 2148-11-186 Cuñado N, Terrones J, Sánchez L, Martínez P, Santos JL (2001) Synaptonemal complex analysis in spermatocytes and oocytes of turbot, Scophthalmus maximus (Pisces, Scophthalmidae). Genome 44:1143-1147. doi:10.1139/gen-44-6-1143 Cuñado N, Terrones J, Sánchez L, Martínez P, Santos JL (2002) Sex- dependent synaptic behaviour in triploid turbot, Scophthalmus 
maximus (Pisces, Scophthalmidae). Hered (Edinb) 89:460-464. doi: 10.1038/sj.hdy.6800165 Danzmann RG, Gharbi K (2001) Gene mapping in fishes: a means to an end. Genetica 111:3-23. doi:10.1023/A:1013713431255 FEAP (2010) Federation of European Aquaculture Producers. Production and Price reports of member associations of the FEAP. http://www. feap.info. Accessed 2 December 2013 Foresti F, Almeida-Toledo LF, Toledo-Filho AS (1981) Polymorphic nature of nucleolus organizer regions in fishes. Cytogenet Cell Genet 31:137-144. doi:10.1159/000131639 Freeman JL, Adeniyi A, Banerjee R, Dallaire S, Maguire SF, Chi J, Ng BL, Zepeda C, Scott CE, Humphray S, Rogers J, Zhou Y, Zon LI, Carter NP, Yang F, Lee C (2007) Definition of the zebrafish genome using flow cytometry and cytogenetic mapping. BMC Genomics 8:

195. doi:10.1186/1471-2164-8-195 García-Cegarra A, Merlo MA, Ponce M, Portela-Bens S, Cross I, Manchado M, Rebordinos L (2013) A preliminary genetic map in Solea senegalensis (Pleuronectiformes, Soleidae) using BAC-FISH and next-generation sequencing. Cytogenet Genome Res 1:227-

240. doi:10.1159/000355001 Gross JB, Protas M, Conrad M, Scheid PE, Vidal O, Jeffery WR, Borowsky R, Tabin CJ (2008) Synteny and candidate gene predic- tion using an anchored linkage map of Astyanax mexicanus. Proc Natl Acad Sci U S A 105:20106-20111. doi:10.1073/pnas. 0806238105 Guyon R, Rakotomanga M, Azzouzi N, Coutanceau JP, Bonillo C, D’Cotta H, Pepey E, Soler L, Rodier-Goud M, D’Hont A, Conte MA, van Bers NE, Penman DJ, Hitte C, Crooijmans RP, Kocher TD, Ozouf-Costaz C, Baroiller JF, Galibert F (2012) A high-resolution map of the Nile tilapia genome: a resource for studying cichlids and other percomorphs. BMC Genomics 13:222. doi:10.1186/1471-2164-13-222 Hermida M, Bouza C, Fernández C, Sciara AA, Rodríguez-Ramilo ST, Fernández J, Martínez P (2013a) Compilation of mapping resources in turbot (Scophthalmus maximus): a new integrated consensus genetic map. Aquaculture 414-415:19-25. doi:10.1016/j. aquaculture.2013.07.040 Hermida M, Rodríguez-Ramilo ST, Hachero-Cruzado I, Herrera M, Sciara AA, Bouza C, Fernández J, Martínez P (2013b) First genetic linkage map for comparative mapping and QTL screening of brill (Scophthalmus rhombus). Aquaculture. doi:10.1016/j.aquaculture. 2013.02.041 Jeukens J, Boyle B, Kukavica-Ibrulj I, St-Cyr J, Lévesque RC, Bernatchez L (2011) BAC library construction, screening and clone sequencing of lake whitefish (Coregonus clupeaformis, Salmonidae) towards the elucidation of adaptive species divergence. Mol Ecol Resour 11:541-549. doi:10.1111/j.1755-0998.2011.02982.x Kai W, Kikuchi K, Tohari S, Chew AK, Tai A, Fujiwara A, Hosoya S, Suetake H, Naruse K, Brenner S, Suzuki Y, Venkatesh B (2011) Integration of the genetic map and genome assembly of fugu facili- tates insights into distinct features of genome evolution in Teleosts and mammals. Genome Biol Evol 3:424-442. doi:10.1093/gbe/ evr041 Kuhl H, Beck A, Wozniak G, Canario AV, Volckaert FA, Reinhardt R (2010) The European sea bass Dicentrarchus labrax genome puzzle: comparative BAC-mapping and low coverage shotgun sequencing. BMC Genomics 11:68. doi:10.1186/1471-2164-11-68 Kuhl H, Tine M, Beck A, Timmermann B, Kodira C, Reinhardt R (2011) Directed sequencing and annotation of three Dicentrarchus labrax L. chromosomes by applying Sanger- and pyrosequencing technol- ogies on pooled DNA of comparatively mapped BAC clones. Genomics 98:202-212. doi:10.1016/j.ygeno.2011.06.004 Lei JL, Liu XF (2011) Culture of turbot: Chinese perspective. In: Daniels HV, Watanabe WO (ed) Practical Flatfish Culture and Stock Enhancement. Wiley- Blackwell, pp185-202 Lorenz S, BrennaHansen S, Moen T, Roseth A, Davidson WS, Omholt SW, Lien S (2010) BAC-based upgrading and physical integration

of a genetic SNP map in Atlantic salmon. Anim Genet 41:48-54. doi:10.1111/j.1365-2052.2009.01963.x Luo MC, Xu K, Ma Y, Deal KR, Nicolet CM, Dvorak J (2009) A high- throughput strategy for screening of bacterial artificial chromosome libraries and anchoring of clones on a genetic map constructed with single nucleotide polymorphisms. BMC Genomics 10:28. doi:10. 1186/1471-2164-10-28 Martínez P, Hermida M, Pardo BG, Fernández C, Castro J, Cal RM, Álvarez-Dios JA, Gómez-Tato A, Bouza C (2008) Centromere- linkage in the turbot (Scophthalmus maximus) through half-tetrad analysis in diploid meiogynogenetics. Aquaculture 280:81-88. doi: 10.1016/j.aquaculture.2008.05.011 Martínez P, Bouza C, Hermida M, Fernández J, Toro MA, Vera M, Pardo B, Millán A, Fernández C, Vilas R, Viñas A, Sánchez L, Felip A, Piferrer F, Ferreiro I, Cabaleiro S (2009) Identification of the major sex-determining region of turbot (Scophthalmus maximus). Genetics 183:1443-1452. doi:10.1534/genetics.109.107979 Maughan PJ, T urner TB, Coleman CE, Elzinga DB, Jellen EN, Morales JA, Udall JA, Fairbanks DJ, Bonifacio A (2009) Characterization of Salt Overly Sensitive 1 (SOS1) gene homoeologs in quinoa (Chenopodium quinoa Willd.). Genome 52:647-657. doi:10.1139/ G09-041 Mazzuchelli J, Kocher TD, Yang F, Martins C (2012) Integrating cytogenetics and genomics in comparative evolutionary studies of cich- lid fish. BMC Genomics 13:463. doi:10.1186/1471-2164-13-46 Molina-Luzón MJ, López JR, Navajas-Pérez R, Robles F, Ruiz-Rejón C, de la Herrán R (2012) Validation and comparison of microsatellite markers derived from Senegalese sole (Solea senegalensis, Kaup) genomic and expressed sequence tags libraries. Mol Ecol Res 12: 956-966. doi:10.1111/j.1755-0998.2012.03163.x Monaco AP, Larin Z (1994) YACs, BACs, PACs and MACs: artificial chromosomes as research tools. Trends Biotechnol 12:280-286. doi: 10.1016/01677799(94)90140-6 Ninwichian P, Peatman E, Liu H, Kucuktas H, Somridhivej B, Liu S, Li P, Jiang Y, Sha Z, Kaltenboeck L, Abernathy JW, Wang W, Chen F, Lee Y, Wong L, Wang S, Lu J, Liu Z (2012) Second-generation genetic linkage map of catfish and its integration with the BAC- based physical map. Bethesda 2:1233-1241. doi:10.1534/g3.112.003962 Oliveira C, Almeida-Toledo LF, Foresti F (2007) Karyotypic evolution in Neotropical fishes. In Pisano E, Ozouf-Costaz C, Foresti F, Kappor BG (ed). Fish cytogenetics. Enfield, pp 111-164 Palti Y, Genet C, Luo MC, Charlet A, Gao G, Hu Y, Castaño-Sánchez C, Tabet-Canale K, Krieg F, Yao J, Vallejo RL, Rexroad CE (2011) A first generation integrated map of the rainbow trout genome. BMC Genomics 12:180. doi:10.1186/1471-2164-12-180 Pardo BG, Bouza C, Castro J, Martínez P, Sánchez L (2001) Localization of ribosomal genes in Pleuronectiformes using Ag-, CMA3-banding and in situ hybridization. Hered (Edinb) 86:531-536. doi:10.1046/j. 1365-2540.2001.00802.x Paux E, Legeai F, Guilhot N, Adam-Blondon AF, Alaux M, Salse J, Sourdille P, Leroy P, Feuillet C (2008) Physical mapping in large genomes: accelerating anchoring of BAC contigs to genetic maps through in silico analysis. Funct Integr Genomics 8:29-32. doi:10. 1007/s10142-007-0068-1 Phillips RB, Amores A, Morasch MR, Wilson C, Postlethwait JH (2006a) Assignment of zebrafish genetic linkage groups to chromosomes. Cytogenet Genome Res 114:155-162. doi:10.1159/000093332 Phillips RB, Nichols KM, DeKoning JJ, Morasch MR, Keatley KA, Rexroad C 3rd, Gahr SA, Danzmann RG, Drew RE, Thorgaard GH (2006b) Assignment of rainbow trout linkage groups to specific chromosomes. Genetics 174:1661-1670. doi:10.1534/genetics.105. 055269 Phillips RB, Keatley KA, Morasch MR, Ventura AB, Lubieniecki KP, Koop BF, Danzmann RG, Davidson WS (2009) Assignment of Atlantic salmon (Salmo salar) linkage groups to specific 
chromosomes: conservation of large syntenic blocks corresponding to whole chromosome arms in rainbow trout (Oncorhynchus mykiss). BMC Genet 10:46. doi:10.1186/1471-2156-10-46 Phillips RB, Park LK, Naish KA (2013) Assignment of Chinook Salmon (Oncorhynchus tshawytscha) linkage groups to specific chromo- somes reveals a karyotype with multiple rearrangements of the chromosome arms of rainbow trout (Oncorhynchus mykiss). Gene Genomes Genet. 3:2289-2295. doi:10.1534/g3.113.008078 Pinkel D, Straume T, Gray JW (1986) Cytogenetic analysis using quan- titative, highsensitivity, fluorescence hybridization. Proc Natl Acad Sci 83:2934-2938 Rodríguez-Ramilo ST, Toro MA, Bouza C, Hermida M, Pardo BG, Cabaleiro S, Martínez P, Fernández J (2011) QTL detection for Aeromonas salmonicida resistance related traits in turbot (Scophthalmus maximus). BMC Genomics 12:541. doi:10.1186/ 1471-2164-12-541 Rodríguez-Ramilo ST, De La Herrán R, Ruiz-Rejón C, Hermida M, Fernández C, Pereiro P, Figueras A, Bouza C, Toro MA, Martínez P, Fernández J (2013a) Identification of quantitative trait loci asso- ciated with resistance to viral haemorrhagic septicaemia (VHS) in turbot (Scophthalmus maximus): a comparison between bacterium, parasite and virus diseases. Mar Biotechnol (NY). doi:10.1007/ s10126-013-9544-X Rodríguez-Ramilo ST, Fernández J, Toro MA, Bouza C, Hermida M, Fernández C, Pardo BG, Cabaleiro S, Martínez P (2013b) Uncovering QTL for resistance and survival time to Philasterides dicentrarchi in turbot (Scophthalmus maximus). Anim Genet 44: 149-157. doi:10.1111/j.13652052.2012.02385.x Ross JA, Peichel CL (2008) Molecular cytogenetic evidence of rearrange- ments on the Y chromosome of the threespine stickleback fish. Genetics 179:2173-2182. doi:10.1534/genetics.108.088559 Ruan X, Wang W, Kong J, Yu F, Huang X (2010) Genetic linkage mapping of turbot (Scophthalmus maximus L.) using microsatellite markers and its application in QT L analysis. Aquaculture 308:89-

100. doi:10.1016/j.aquaculture.2010.08.010 Sánchez-Molano E, Cerna A, Toro MA, Bouza C, Hermida M, Pardo BG, Cabaleiro S, Fernández J, Martínez P (2011) Detection of growth- related QTL in turbot (Scophthalmus maximus). BMC Genomics 12:

473. doi:10.1186/1471-2164-12-473 Sasaki T, Shimizu A, Ishikawa SK, Imai S, Asakawa S, Murayama Y, Khorasani MZ, Mitani H, Furutani-Seiki M, Kondoh H, Nanda I, Schmid M, Schartl M, Nonaka M, Takeda H, Hori H, Himmelbauer H, Shima A, Shimizu N (2007) The DNA sequence of medaka chromosome LG22. Genomics 89:124-133. doi:10.1016/j.ygeno. 2006.09.003 Shao CW, Chen SL, Scheuring CF, Xu JY, Sha ZX, Dong XL, Zhang HB (2010) Construction of two BAC libraries from half-smooth tongue sole Cynoglossus semilaevis and identification of clones containing candidate sexdetermination genes. Mar Biotechnol (NY) 12:558-

568. doi:10.1007/s10126-009-9242-x Shimoda N, Knapik EW, Ziniti J, Sim C, Yamada E, Kaplan S, Jackson D, de Sauvage F, Jacob H, Fishman MC (1999) Zebrafish genetic map with 2000 microsatellite markers. Genomics 58:219-232. doi: 10.1006/geno.1999.5824 Urton JR, McCann SR, Peichel CL (2011) Karyotype differentiation between two stickleback species (Gasterosteidae). Cytogenet Genome Res 135:150-159. doi:10.1159/000331232

Viñas A, Taboada X, Vale L, Robledo D, Hermida M, Vera M, Martínez P (2012) Mapping of DNA sex-specific markers and genes related to sex differentiation in turbot (Scophthalmus maximus). Mar Biotechnol (NY) 14:655-663. doi:10.1007/s10126-012-9451-6 Wang S, Xu P, Thorsen J, Zhu B, de Jong PJ, Waldbieser G, Kucuktas H, Liu Z (2007) Characterization of a BAC library from channel catfish Ictalurus punctatus: indications of high levels of chromosomal reshuffling among teleost genomes. Mar Biotechnol (NY) 9:701-

711. doi:10.1007/s10126-007-9021-5 Wang CM, Lo LC, Feng F, Gong P, Li J, Zhu ZY, Lin G, Yue GH (2008) Construction of a BAC library and mapping BAC clones to the linkage map of Barramundi, Lates calcarifer. BMC Genomics 9:

139. doi:10.1186/1471-2164-9-139 Wang X, Zhang Q, Ren J, Jiang Z, Wang C, Zhuang W, Zhai T (2009) The preparation of sex-chromosome-specific painting probes and construction of sex chromosome DNA library in half-smooth tongue sole (Cynoglossus semilaevis). Aquaculture 297:78-84. doi:10. 1016/j. aquaculture .2009.09.020 Xia JH, Feng F, Lin G, Wang CM, Yue GH (2010) A first generation BAC-based physical map of the Asian seabass (Lates calcarifer). PLoS One 5:e11974. doi:10.1371/journal. pone.0011974 Xu P, Wang S, Liu L, Peatman E, Somridhivej B, Thimmapuram J, Gong G, Liu Z (2006) Channel catfish BAC-end sequences for marker development and assessment of syntenic conservation with other fish species. Anim Genet 37:321326. doi:10.1111/j.1365-2052. 2006.01453.x Xu P, Wang S, Liu L, Thorsen J, Kucuktas H, Liu Z (2007) A BAC-based physical map of the channel catfish genome. Genomics 90:380-388. doi:10.1016/j.ygeno.2007.05.008 Xu P, Li J, Li Y, Cui R, Wang J, Wang J, Zhang Y, Zhao Z, Sun X (2011) Genomic insight into the common carp (Cyprinus carpio) genome by sequencing analysis of BAC-end sequences. BMC Genomics 12:

188. doi:10.1186/1471-2164-12-188 Yim YS, Moak P, Sanchez-Villeda H, Musket T A, Close P, Klein PE, Mullet JE, McMullen MD, Fang Z, Schaeffer ML, Gardiner JM, Coe EH Jr, Davis GL (2007) A BAC pooling strategy combined with PCR-based screenings in a large, highly repetitive genome enables integration of the maize genetic and physical maps. BMC Genomics 8:47. doi:10. 1186/1471-2164-8-47 You FM, Luo MC, Xu K, Deal KR, Anderson OD, Dvorak J (2010) A new implementation of high-throughput five-dimensional clone pooling strategy for BAC library screening. BMC Genomics 11:

692. doi:10.1186/1471-2164-11-692 Zhang Y, Zhang X, Scheuring CF, Zhang HB, Huan P, Li F, Xiang J (2008) Construction and characterization of two bacterial artificial chromosome libraries of Zhikong scallop, Chlamys farreri Jones et Preston, and identification of BAC clones containing the genes involved in its innate immune system. Mar Biotechnol (NY) 10:358-365. doi:10.1007/ s10126-007-9071-8 Zhao L, Zhang Y, Ji P, Zhang X, Zhao Z, Hou G, Huo L, Liu G, Li C, Xu P, Sun X (2013) A dense genetic linkage map for common carp and its integration with a BAC-based physical map. PLoS One 8: e63928. doi:10.1371/journal.pone.0063928 
